\title{
SUBSTRATE INHIBITION IN LIPASE-CATALYSED TRANSESTERIFICATION OF MANDELIC ACID WITH VINYL ACETATE
}

\author{
Katarzyna Dąbkowska* ${ }^{*}$ Maciej Pilarek, Krzysztof W. Szewczyk \\ Warsaw University of Technology, Faculty of Chemical and Process Engineering, Department of \\ Biotechnology and Bioprocess Engineering, Waryńskiego 1, 00-645 Warszawa, Poland
}

\begin{abstract}
Kinetic resolution of $(R)$ - and $(S)$-mandelic acid by its transesterification with vinyl acetate catalysed by Burholderia cepacia lipase has been studied. The influence of the initial substrate concentration on the kinetics of process has been investigated. A modified ping-pong bi-bi model of enzymatic transesterification of $(S)$-mandelic acid including substrate inhibition has been developed. The values of kinetic parameters of the model have been estimated. We have shown that the inhibition effect revealed over a certain threshold limit value of the initial concentration of substrate.
\end{abstract}

Keywords: kinetic resolution, enantiomers, kinetic model, lipase, substrate inhibition

\section{INTRODUCTION}

The ability of lipases to catalyse many various enzymatic reactions has attracted increasing interest throughout the world. In non-aqueous media they catalyse a wide spectrum of reactions such as: alcoholysis, transesterification and ester synthesis or regiospecific acylation (Sharma et al., 2011; Szewczyk et al., 2001). Lipase-catalysed transesterification is one of the commonly applied methods for kinetic resolution of enantiomers in organic media (Ghanem and Aboul-Enein, 2005). This technique is based on differences in the transformation rate of particular enantiomers with a given acylating agent. Production of enantiomerically enriched compounds has recently become a major research area especially in pharmaceutical and fine-chemical industry. The requirement to prepare pure enantiomers arises from totally different pharmacological activities of enantiomeric pairs (Maier et al., 2001; Stinson, 2001).

Pure enantiomers of mandelic acid and their derivatives are recognised as important chiral building blocks, auxiliaries and resolving agents in pharmacochemistry and enzymatic bioprocesses (Kibara et al., 1996; Survivet and Vatèle, 1998; Terreni et al., 2001). Several reports of mandelic acid enantiomers production have been presented so far (Gröger, 2001; Palomo et al. 2002; Yadav and Sivakumar, 2004). One of the easiest and the most promising methods seems to be a kinetic resolution of mandelic acid racemates by lipase-catalysed transesterification (Campbell et al., 2003; Qeiroz and Nascimento, 2002). In our previous papers (Dąbkowska and Szewczyk, 2007; Dąbkowska and Szewczyk, 2009) we have shown that $B$. cepacia lipase is highly enantioselective towards $(S)$-substrate during transesterification with vinyl acetate in isopropyl ether used as the solvent. At $25^{\circ} \mathrm{C}$ only the $(S)$ mandelic acid form was transformed into the product, whilst the conversion of $(R)$-mandelic acid has 
not been observed. The simple ping-pong bi-bi model has been used to describe the reaction kinetics (Dąbkowska and Szewczyk, 2007).

The aims of our present work were to investigate the influence of the initial substrate concentration on the rate of transesterification of $(S)$-mandelic acid catalysed by B. cepacia lipase and to discuss substrate inhibition in the studied enzymatic reaction. An improved ping-pong bi-bi kinetic model has been used to explicitly determine substrate effects on lipase. To our knowledge this is the first attempt to apply substrate inhibition to completely define the mechanism of mandelic acid transesterification in general.

\section{MATERIALS AND METHODS}

\subsection{Enzyme}

Lipase from Burkholderia cepacia was purchased from Amano Pharmaceutical Co. (Japan) as Amano PS lipolytic enzyme preparation. It contains enzyme concentrate and diatomaceous earth in the ratio of 50:50 (w/w). The enzyme concentrate could also contain dextrin and some solid constituents originating from the fermentation media. The water and protein content was $25 \mathrm{mg} / \mathrm{g}$ and $65 \mathrm{mg} / \mathrm{g}$, respectively. According to the Certificate of Analysis supplied by the producer the activity of enzyme was $31600 \mathrm{U} / \mathrm{g}$.

\subsection{Chemicals}

All the chemicals which were used in the experiments as well as all HPLC solvents were of analytical grade. $(R, S)$-mandelic acid $(\geq 98 \%)$, trifluoroacetic acid $(\geq 98 \%)$ and vinyl acetate $(\geq 99 \%)$ were purchased from Sigma-Aldrich whilst $n$-hexane (99\%), isopropyl ether (98\%) and ethanol $(99.8 \%)$ were obtained from POCH S.A. (Gliwice, Poland).

\subsection{Analytical methods}

The concentration of mandelic acid and O-acetylmandelic acid enantiomers was determined by HPLC method. The Varian 65 CL System chromatograph (Varian, USA) supported with Chiralcel OD column (1. $250 \mathrm{~mm}$, i.d. $4.6 \mathrm{~mm}$ ) from Daicel Chemical Industries, Ltd. (Japan) and with UV detection at 228 $\mathrm{nm}$ was used. The mixture composed of $n$-hexane, ethanol and trifluoroacetic acid at the volumetric ratio of 95:5:0.5 respectively was applied as a mobile phase. The analysis was performed at a temperature of $4^{\circ} \mathrm{C}$, whilst the flow rate of the mobile phase was set at $1.0 \mathrm{~cm}^{3} / \mathrm{min}$.

The retention times were determined as follows: $22.0 \mathrm{~min}$. for $(R)$-mandelic acid, $19.5 \mathrm{~min}$. for $(S)$ mandelic acid, $12.5 \mathrm{~min}$. for $(R)$-O-acetylmandelic acid and $11.5 \mathrm{~min}$. for $(S)$-O-acetylmandelic acid.

\subsection{Numerical methods}

The values of kinetic parameters were estimated numerically by minimising the total error $(\varepsilon)$ between measured $(j)$ and calculated ( $c a l)$ values of $(S)$-mandelic acid concentrations $\left(C_{M A}\right)$, according to Equation 1:

$$
\varepsilon=\sum_{j}\left(C_{M A, j}-C_{M A, c a l}\right)^{2}
$$

A fourth order Runge-Kutta method was used to solve the model equations. 


\subsection{Transesterification of mandelic acid with vinyl acetate}

A kinetic resolution of $(R)$ - and $(S)$-mandelic acid isomers by lipase catalysed transesterification with vinyl acetate was performed in $100 \mathrm{~cm}^{3}$ Erlenmeyer flasks thermostated in a water bath shaker $\left(T=25{ }^{\circ} \mathrm{C}, 150 \mathrm{rpm}\right)$. The reaction mixtures were prepared by dissolving an appropriate amount of $(R, S)$-mandelic acid and vinyl acetate in $75 \mathrm{~cm}^{3}$ of isopropyl ether. Then $300 \mathrm{mg}$ of B. cepacia lipase (Amano PS) was added to initiate enzymatic process. Depending on the experiment, the initial concentration of each mandelic acid enantiomer in reaction mixture equalled to $0.004,0.015,0.025$ or $0.035 \mathrm{~mol} / \mathrm{dm}^{3}$ (i.e. it corresponded to 2 much higher concentration of racemic mandelic acid in every studied case). Vinyl acetate was used at a 10-fold molar excess to racemic mandelic acid. The $0.2 \mathrm{~cm}^{3}$ samples of reaction mixture were harvested at appropriate time intervals, then they were filtered with single-use syringe filters $(0.2 \mu \mathrm{m}$; polypropylene housing, nylon membrane) and finally diluted (1:10) with isopropyl ether prior to HPLC analysis.

\section{RESULTS AND DISCUSSION}

\subsection{Effect of initial mandelic acid concentration on transesterification progress}

The conversion profiles of $(S)$-mandelic acid transesterification for various initial concentration of substrate have been presented in Figure 1. It can be easily seen that the studied reaction proceeded faster in the case of lower values of the initial mandelic acid concentration. However, no significant differences in the reaction rates were observed for substrate concentrations not exceeding 0.015 $\mathrm{mol} / \mathrm{dm}^{3}$.

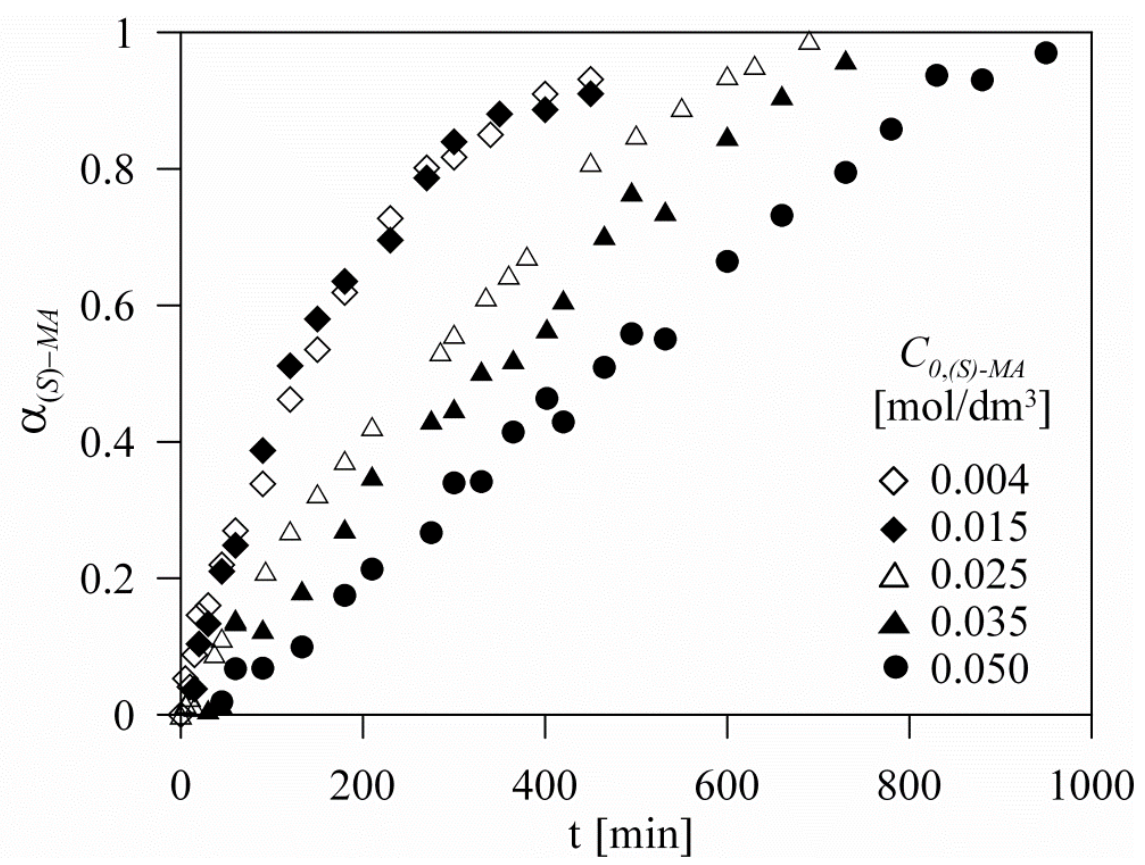

Fig. 1. The influence of the mandelic acid initial concentration on conversion profiles of $(S)$-mandelic acid

According to the simple ping-pong bi-bi model, which has been used previously to describe the transesterification progress of (S)-mandelic acid (Dąbkowska and Szewczyk 2007), the rate of the reaction can be expressed as follows: 


$$
r=\frac{r_{\max }}{1+\frac{C_{V A}}{K_{V A}}+\frac{C_{(S)-M A}}{K_{(S)-M A}}}
$$

In our experiments the molar ratio of vinyl acetate to $(S)$-mandelic acid equalled 20:1. In such case the overall concentration of vinyl acetate can be described as:

$$
C_{V A}=20 \cdot C_{0,(S)-M A}-\left(C_{0,(S)-M A}-C_{(S)-M A}\right)
$$

Taking into account Equation 3, the integration of Equation 2 for the initial conditions (at $t=0$ $\left.C_{(S)-M A}=C_{0,(S)-M A}\right)$ gives the following expression:

$$
t\left(\alpha_{M A}\right)=\frac{1}{r_{\max }}\left[\alpha_{M A} C_{0,(S)-M A}-K_{V A} \ln \left(\frac{20-\alpha_{M A}}{20}\right)-K_{(S)-M A} \ln \left(1-\alpha_{M A}\right)\right]
$$

where $\alpha_{M A}$ is the conversion of racemic mandelic acid, defined as:

$$
\alpha_{M A}=\frac{C_{0, M A}-C_{M A}}{C_{0, M A}}
$$

Only one enantiomer of mandelic acid undergoes the reaction under the applied conditions, thus $\alpha_{M A} \in\langle 0 \div 0.5\rangle$ and it can be assumed that:

$$
\ln \left(\frac{20-\alpha_{M A}}{20}\right) \approx 0
$$

Additionally, Taylor series expansion gives:

$$
\ln \left(1-\alpha_{M A}\right) \approx \alpha_{M A}
$$

Finally, from Equations 4, 6 and 7 the $\alpha_{M A}$ can be defined as:

$$
\alpha_{M A}=\frac{r_{\max } t}{C_{0,(S)-M A}+K_{(S)-M A}}
$$

As we previously published (Dąbkowska and Szewczyk, 2009) the enzyme-(S)-mandelic acid affinity constant, $K_{(S)-M A}$, equals $0.99 \mathrm{~mol} / \mathrm{dm}^{3}$. Therefore, for $C_{0,(S)-M A}$ much lower than $K_{(S)-M A}$ value, the conversion of mandelic acid for ping-pong bi-bi mechanism should be practically independent of the initial mandelic acid concentration in the reaction mixture. However, the results of our present study, showed in Figure 1, are not in agreement with the model predictions discussed above.

Table 1. Estimated kinetic parameters for ping-pong bi-bi mechanism

\begin{tabular}{|c|c|c|c|c|c|}
\hline $\begin{array}{c}C_{O,(S)-M A} \\
\mathrm{~mol} / \mathrm{dm}^{3}\end{array}$ & $\begin{array}{c}C_{O, V A} \\
\mathrm{~mol} / \mathrm{dm}^{3}\end{array}$ & $\begin{array}{c}r_{\max } \cdot 10^{3} \\
\mathrm{~mol} / \mathrm{dm}^{3} \cdot \mathrm{min}\end{array}$ & $\begin{array}{c}K_{(S)-M A} \\
\mathrm{~mol} / \mathrm{dm}^{3}\end{array}$ & $\begin{array}{c}K_{V A} \\
\mathrm{~mol} / \mathrm{dm}^{3}\end{array}$ & $\begin{array}{c}\text { relative } \\
\text { error } \%\end{array}$ \\
\hline 0.004 & 0.08 & 6.54 & 0.98 & 6.13 & 8.66 \\
\hline 0.015 & 0.30 & 6.48 & 0.99 & 6.02 & 3.30 \\
\hline 0.025 & 0.50 & 5.41 & 0.67 & 34.66 & 9.82 \\
\hline 0.035 & 0.70 & 4.33 & 0.43 & 42.91 & 7.24 \\
\hline 0.050 & 1.00 & 3.19 & 0.04 & 55.82 & 10.81 \\
\hline
\end{tabular}

The estimated values of the simple ping-pong bi-bi model parameters for various initial mandelic acid concentrations are summarised in Table 1 . In the case of a reaction carried out with $C_{0,(S)-M A}=0.004$ and 
$0.015 \mathrm{~mol} / \mathrm{dm}^{3}$ all the kinetic parameters are independent of the initial substrate concentrations. In the case of higher initial concentrations of the substrate, the maximum reaction rate and the enzymemandelic acid affinity constant decrease, but the enzyme-vinyl acetate affinity constant increases with the increase of initial substrate concentration.

Based on the dependence of the presented ping-pong bi-bi kinetic parameters on the initial composition of reaction mixture we hypothesised the presence of an effect which triggered a reduction of the overall reaction rate at relatively high mandelic acid concentrations.

\subsection{Ping-pong bi-bi model with substrate inhibition}

In our next investigations several attempts were made to describe more accurately the kinetics of the studied reaction and a few modifications of simple ping-pong kinetic mechanism were considered. An incorporation of competitive inhibition by acyl acceptor, as the most commonly reported pathway of inhibition for lipase-catalysed reactions (Pilarek and Szewczyk, 2007; Segel, 1993), as well as product inhibition and enzyme deactivation by vinyl alcohol did not produce satisfactory results and did not fit well with our experimental data.

Here we propose a modified ping-pong bi-bi kinetics including substrate inhibition of enzyme by mandelic acid to describe accurately the effects of the initial substrate influence on lipase activity observed in the presented experiments. Such an attempt assumes that in the case of a relatively high substrate concentration in reaction mixture, two molecules of $(S)$-mandelic acid can simultaneously bind to the enzyme active site. Finally, a triple enzyme-substrate-substrate complex is formed which is unable to further convert into the product. According to our considerations, the rate of $(S)$-mandelic acid conversion can be defined as:

$$
r=\frac{r_{\max }}{1+\frac{C_{V A}}{K_{V A}}+\frac{C_{(S)-M A}}{K_{(S)-M A}}+\frac{C_{(S)-M A} \cdot K_{(S)-M A}}{K_{i,(S)-M A}}}
$$

The estimated values of kinetic parameters for modified ping-pong bi-bi with the substrate inhibition have been showed in Table 2. As can be easily seen all the kinetic parameters are independent of the initial concentration of the substrate.

Table 2. Values of kinetic parameters estimated for ping-pong bi-bi kinetics with substrate inhibition by mandelic acid

\begin{tabular}{|c|c|c|c|c|c|c|}
\hline $\begin{array}{c}C_{C_{,(S)}-M A} \\
\mathrm{~mol} / \mathrm{dm}^{3}\end{array}$ & $\begin{array}{c}C_{0, V A} \\
\mathrm{~mol} / \mathrm{dm}^{3}\end{array}$ & $\begin{array}{c}r_{\max } \cdot 10^{3} \\
\mathrm{~mol} / \mathrm{dm}^{3} \cdot \mathrm{min}\end{array}$ & $\begin{array}{c}K_{(S)-M A} \\
\mathrm{~mol} / \mathrm{dm}^{3}\end{array}$ & $\begin{array}{c}K_{V A} \\
\mathrm{~mol} / \mathrm{dm}^{3}\end{array}$ & $\begin{array}{c}K_{i,(S)-M A} \cdot 10^{4} \\
\mathrm{~mol} / \mathrm{dm}^{3}\end{array}$ & $\begin{array}{c}\text { relative } \\
\text { error \% }\end{array}$ \\
\hline 0.004 & 0.08 & 6.42 & 0.98 & 5.98 & 5.03 & 6.18 \\
\hline 0.015 & 0.30 & 6.78 & 0.94 & 5.64 & 5.99 & 3.66 \\
\hline 0.025 & 0.50 & 6.46 & 1.01 & 6.07 & 3.13 & 9.14 \\
\hline 0.035 & 0.70 & 6.31 & 1.00 & 6.12 & 4.03 & 6.28 \\
\hline 0.050 & 1.00 & 6.51 & 0.98 & 6.11 & 3.75 & 5.72 \\
\hline \multicolumn{2}{|c|}{ mean } & 6.50 & 0.98 & 5.98 & 4.39 & \\
\hline
\end{tabular}

A comparison of experimental data and the modified ping-pong bi-bi model curves have been showed in Figure 2. All the mathematical predictions fitted well the experimental data over the entire range of the initial mandelic acid concentrations used in the tests. 


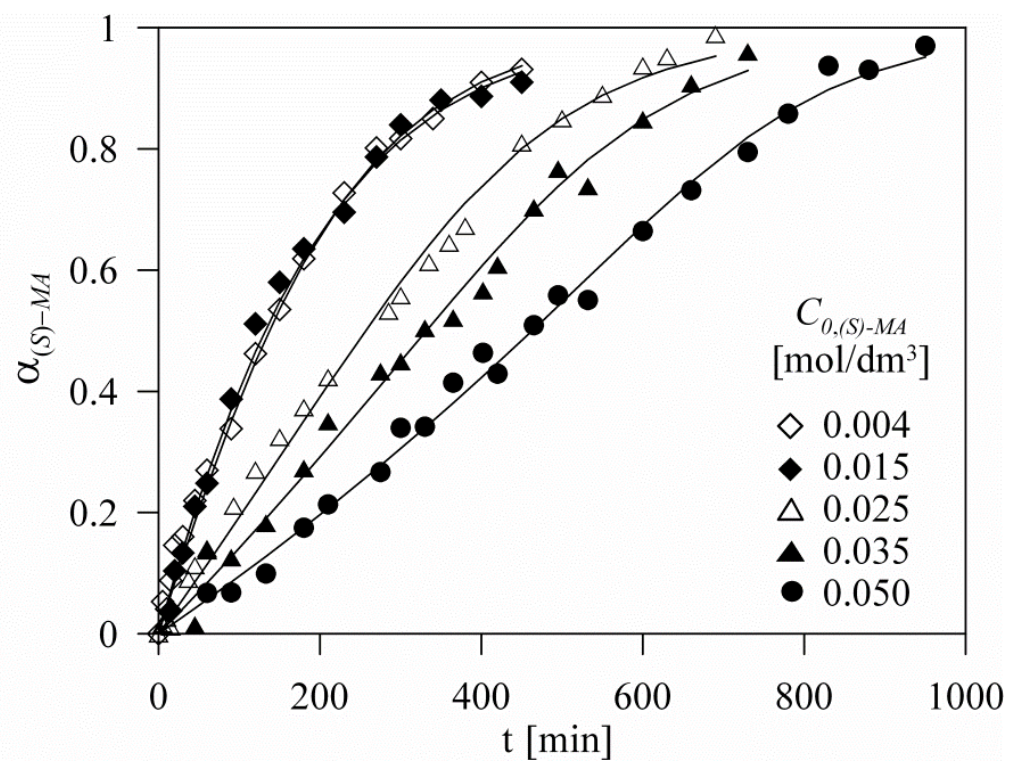

Fig. 2. A comparison of experimental data (dots) and the model curves predicted by modified ping-pong bi-bi kinetic model with enzyme inhibition by mandelic acid (continuous lines)

\subsection{Threshold limit values of initial (S)-mandelic acid concentration}

Results of the numerical simulation (based on Equation 9) of (S)-mandelic acid transesterification rate for various initial concentrations of racemic substrate $\left(C_{0, M A}\right)$ have been presented in Figure 3 . The mean values of the estimated kinetic parameters, taken from Table 2, have been incorporated. It can be seen that the rate of the reaction significantly increased with substrate concentration increase up to reaching the threshold limit point $\left(C_{M A}\right)_{l i m}=0.042 \mathrm{~mol} / \mathrm{dm}^{3}$, and then dropped. In our opinion, for relatively small mandelic acid concentrations, the reaction rate can be considered as practically independent of the initial concentration of the substrate. Based on the presented simulation results of the transesterification process we hypothesise that for the initial concentration of racemic mandelic acid lower than the threshold limit $\left(C_{0, M A}\right)_{\lim }=0.042 \mathrm{~mol} / \mathrm{dm}^{3}$, the simple ping-pong bi-bi mechanism can be applied. Above the $\left(C_{0, M A}\right)_{l i m}$ value the substrate inhibition should be taken into account to fully describe the kinetics of the studied lipase-catalysed transesterification.

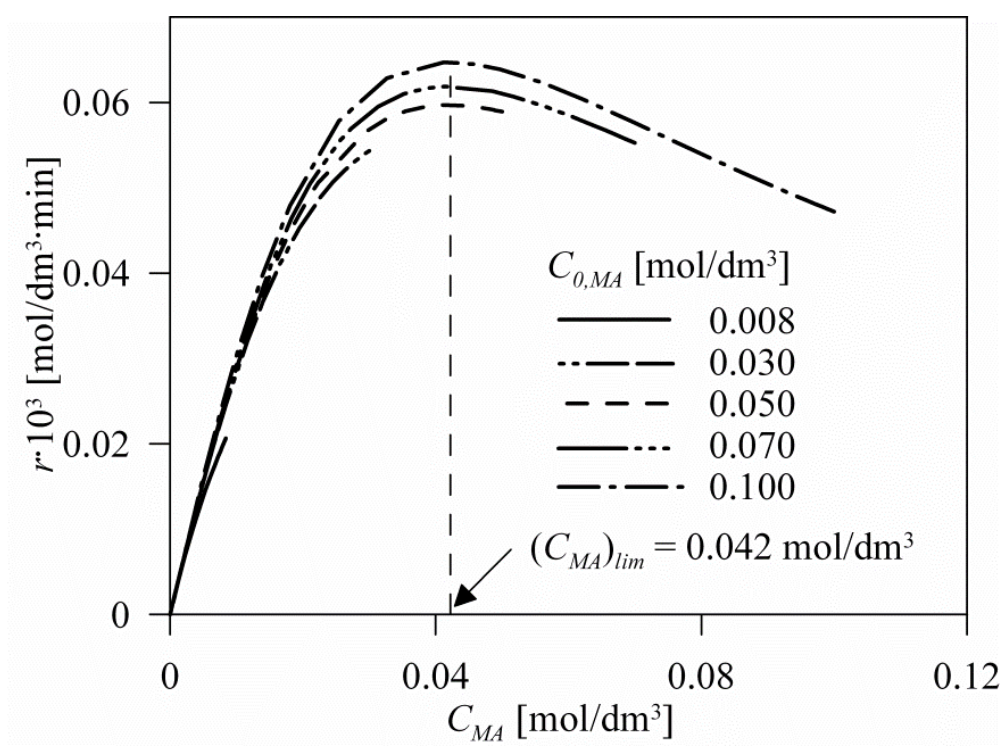

Fig. 3. Results of the numerical simulations of $(S)$-mandelic acid transesterification rate for the modified ping-pong bi-bi kinetics for various initial concentrations of substrate 


\section{CONCLUSIONS}

The influence of the initial concentration of substrate on the rate of kinetic resolution of $(S)$-mandelic acid catalysed by B. cepacia lipase has been discussed in-depth. The modified ping-pong bi-bi mechanism with substrate inhibition of enzyme has been applied to fully describe the progress of mandelic acid transesterification with vinyl acetate. The inhibition effect revealed over the threshold limit value of the initial concentration of substrate, which has been determined as $0.042 \mathrm{~mol} / \mathrm{dm}^{3}$. All the predictions of the reaction kinetics fitted well the experimental data over the entire range of the initial (S)-mandelic acid concentrations. To sum up, we conclude that results of such investigations can be useful for more rational design of industrial systems applied for enantiomerically pure mandelic acid production.

\section{SYMBOLS}

\begin{tabular}{|c|c|}
\hline$C_{0, M A}$ & initial $(R, S)$-mandelic acid concentration, $\mathrm{mol} / \mathrm{dm}^{3}$ \\
\hline$C_{0,(S)-M A}$ & initial $(S)$-mandelic acid concentration, $\mathrm{mol} / \mathrm{dm}^{3}$ \\
\hline$C_{M A}$ & $(R, S)$-mandelic acid concentration, $\mathrm{mol} / \mathrm{dm}^{3}$ \\
\hline$C_{(S)-M A}$ & $(S)$-mandelic acid concentration, $\mathrm{mol} / \mathrm{dm}^{3}$ \\
\hline$\left(C_{M A}\right)_{\lim }$ & threshold limit value of racemic mandelic acid concentration, $\mathrm{mol} / \mathrm{dm}^{3}$ \\
\hline$C_{V A}$ & vinyl acetate concentration, $\mathrm{mol} / \mathrm{dm}^{3}$ \\
\hline$K_{i,(S)-M A}$ & $(S)$-mandelic acid inhibition constant, $\mathrm{mol} / \mathrm{dm}^{3}$ \\
\hline$K_{M A}$ & enzyme-mandelic acid affinity constant, $\mathrm{mol} / \mathrm{dm}^{3}$ \\
\hline$K_{V A}$ & enzyme-vinyl acetate affinity constant, $\mathrm{mol} / \mathrm{dm}^{3}$ \\
\hline$r$ & reaction rate, $\mathrm{mol} /\left(\mathrm{dm}^{3} \mathrm{~min}\right)$ \\
\hline$r_{\max }$ & maximum reaction rate, $\mathrm{mol} /\left(\mathrm{dm}^{3} \mathrm{~min}\right)$ \\
\hline \multicolumn{2}{|c|}{ Greek symbols } \\
\hline$\alpha_{M A}$ & conversion of $(R, S)$ - mandelic acid \\
\hline$\alpha_{(S)-M A}$ & conversion of $(S)$-mandelic acid \\
\hline
\end{tabular}

\section{REFERENCES}

Campbell R.F., Fitzpatric K., Inghardt T., Karlsson O., Nilsson K., Reilly J.E., Yet L., 2003. Enzymatic resolution of substituted mandelic acids. Tetrahedron Lett., 44, 5477-5481. DOI: 10.1016/S0040-4039(03)01270-X.

Dąbkowska K., Szewczyk K.W., 2007. Mathematical modelling of enzymatic transesterification of mandelic acid with vinyl acetate. Chem. Proc. Eng., 28, 795-802.

Dąbkowska K., Szewczyk K.W., 2009. Influence of temperature on the activity and enantioselectivity of Burkholderia cepacia lipase in the kinetic resolution of mandelic acid enantiomers. Biochem. Eng. J., 46, 147153. DOI: 10.1016/j.bej.2009.04.023.

Ghanem A., Aboul-Enein H.Y., 2005. Application of lipases in kinetic resolution of racemates. Chirality, 17, 1-15. DOI: 10.1002/chir.20089.

Gröger H., 2001. Enzymatic Routes to Enantiomerically pure aromatic $\alpha$-hydroxy carboxylic acids: A further example for the diversity of biocatalysis. Adv. Synth. Catal., 343, 547-558. DOI: 10.1002/16154169(200108)343:6/7<547::AID-ADSC547>3.0.CO;2-A.

Kibara K., Sakai K., Hashimoto Y., Nohira H., Saigo K., 1996. Design of resolving reagents: p-substituted mandelic acid as resolving reagents for 1-arylalkylamines. Tetrahedron: Asymmetry, 7, 1539-1542. DOI: 10.1016/0957-4166(96)00175-9.

Maier N.M., Franco P., Lindner W., 2001. Separation of enantiomers: needs, challenges, perspectives. J. Chromatogr. A, 906, 3-33. DOI: 10.1016/S0021-9673(00)00532-X. 
Palomo M., Fernandez-Lorente G., Mateo C., Ortiz C., Guisan J.M., Fernandez-Lafuente R., 2002. Modulation of the enantioselectivity of lipases via controlled immobilization and medium engineering: hydrolytic resolution of mandelic acid esters. Enz. Microb. Technol., 31, 775-783. DOI: 10.1016/S0141-0229(02)00169-2.

Pilarek M., Szewczyk K.W., 2007. Kinetic model of 1,3-specific triacylglycerols alcoholysis catalyzed by lipases. J. Biotechnol., 127, 736-744. DOI: 10.1016/j.jbiotec.2006.08.012.

Segel I.H., 1993. Enzyme kinetic. Behavior and analysis of rapid equilibrium and steady-state enzyme systems. Wiley, New York, 827-828.

Sharma D., Sharma B., Shukla A.K., 2011. Biotechnological approach of microbial lipase: A review. Biotechnology, 10, 23-40. DOI: 10.3923/biotech.2011.23.40.

Stinson C.S., 2001. Chiral pharmaceuticals. Chem. Eng. News, 79, 79-97. DOI: 10.1021/cen-v079n040.p079.

Surivet J.-P., Vatèle J.-M., 1998. First total synthesis of (-)-8-epi-9-deoxygoniopypyrone. Tetrahedron Lett., 39, 9681-9682. DOI: 10.1016/S0040-4039(98)02269-2.

Szewczyk K.W., Pilarek M., Wrona M., 2001. Enzymatic propanolysis of triacetin. Inż. Chem. Proc., 22, 13511356.

Terreni M., Pagani G., Ubiali D., Fernández-Lafuente R., Mateo C., Guisán J.M., 2001. Modulation of penicillin acylase properties via immobilization techniques: one-pot chemoenzymatic synthesis of caphamandole from cephalosporin C. Bioorg. Med. Chem. Lett., 11, 2429-2432. DOI: 10.1016/S0960-894X(01)00463-2.

Queiroz N., Nascimento M. G., 2002. Pseudomonas sp. lipase immobilized in polymers versus the use of free enzyme in the resolution of $(R, S)$-methyl mandelate. Tetrahedron Lett., 43, 5225-5227. DOI: 10.1016/S00404039(02)01057-2.

Yadav G.D., Sivakumar P., 2004. Enzyme-catalysed optical resolution of mandelic acid via $(R, S)$-methyl mandelate in non-aqueous media. Biochem. Eng. J., 19, 101-107. DOI: 10.1016/j.bej.2003.12.004.

Received 16 May 2012

Received in revised form 12 November 2012

Accepted 19 November 2012 